\title{
POR QUE MEDITAR? A EXPERIÊNCIA SUBJETIVA DA PRÁTICA DE MEDITAÇÃO'
}

\author{
Carolina Baptista Menezes \\ Débora Dalbosco Dell'Aglio"
}

\begin{abstract}
RESUMO. A meditação, caracterizada como o treino da atenção plena à consciência do momento presente, tem sido associada a um maior bem-estar mental, emocional e físico. Este estudo investigou como os praticantes da meditação passiva - sentada e silenciosa - percebem o impacto de sua prática no seu cotidiano. Ao todo, 105 participantes os quais frequentavam havia no mínimo um mês algum centro de meditação da cidade de Porto Alegre responderam a uma questão aberta sobre como a meditação se reflete em suas vidas. Através da análise de conteúdo, foram identificadas seis categorias, representando os benefícios citados: cognitivos, emocionais, físicos, espirituais, sociais e outros. Através da regressão de Poisson, foram observados alguns fatores associados às categorias identificadas. Conclui-se que a experiência subjetiva da meditação se reflete de diversas formas, com predominância de benefícios cognitivos e emocionais, e que esta prática pode constituir uma ferramenta para o desenvolvimento psicológico saudável.
\end{abstract}

Palavras-chave: Meditação, mindfulness, qualidade de vida.

\section{WHY MEDITATE? THE SUBJECTIVE EXPERIENCE OF THE MEDITATION PRACTICE}

\begin{abstract}
Meditation, characterized as the training of full attention to the consciousness of the present moment, has been associated with greater mental, emotional and physical well-being. This study aimed at investigating how practitioners of the passive meditation - silent and sitting - perceive the impact of their training on their daily lives. A total of 105 participants, whom had been going to a meditation center in the city of Porto Alegre for at least one month, answered an open question investigating how meditation reflects on their lives. Through content analysis six categories representing the effects of meditation were identified: cognitive, emotional, physical, spiritual, social and others. Through Poisson regression some factors associated with the categories identified were observed. It has been concluded that the subjective experience of meditation can influence life in many ways, especially concerning cognitive and emotional benefits, and that this practice could be a tool for a healthy psychological development.
\end{abstract}

Key words: Meditation, mindfulness, quality of life.

\section{¿POR QUÉ MEDITAR? LA EXPERIENCIA SUBJETIVA DE LA PRÁCTICA DE MEDITACIÓN}

RESUMEN. La meditación, caracterizada como el entrenamiento de la atención llena a la conciencia del momento presente, ha sido asociada a un mayor bienestar mental, emocional y físico. Este estudio investigó como los practicantes de la meditación del tipo pasiva - sentada y silenciosa - perciben el impacto de su práctica en su cotidiano. Un total de 105 participantes, que frecuentaban hace como mínimo un mes algún centro de meditación de la ciudad de Porto Alegre, respondieron a una cuestión abierta sobre como la meditación se refleja en sus vidas. A través del análisis de contenido, fueron identificadas seis categorías representando los beneficios citados: cognitivos, emocionales, físicos, espirituales, sociales y otros. A través de la regresión de Poisson, fueron observados algunos factores asociados a las categorías identificadas. Fue posible concluir que la experiencia subjetiva de la meditación se refleja de diversas formas, con predominancia de beneficios cognitivos y emocionales, y que esta práctica puede constituir una herramienta para el desarrollo psicológico saludable.

Palabras-clave: Meditación, mindfulness, cualidad de vida.

\footnotetext{
1 Apoio: $\mathrm{CNPq}$.

* Mestre em Psicologia. Doutoranda em Psicologia com bolsa CNPq, Programa de Pós-Graduação em Psicologia, Universidade Federal do Rio Grande do Sul.

\# Professora e Pesquisadora do Programa de Pós-Graduação em Psicologia, coordenadora do Núcleo de Estudos e Pesquisas em Adolescentes (NEPA), Instituto de Psicologia, Universidade Federal do Rio Grande do Sul.
} 
A meditação, caracterizada como o treino da atenção plena à consciência do momento presente, tem sido associada a um maior bem-estar físico, mental e emocional (Shapiro, Schwartz, \& Santerre, 2005). Esta prática teve origem nas filosofias espirituais do Oriente, mas especialmente a partir da década de 60 o movimento de trazê-la para o Ocidente ganhou força. Assim, juntamente com a crescente busca pela prática pessoal, também houve um maior interesse científico por ela. Desde então, uma das áreas de maior interface com a meditação e as filosofias relacionadas é a psicologia. A meditação já foi bastante referida e estudada por psicanalistas como Erich Fromm e Karen Horney, sendo associada e comparada à escuta flutuante que o psicanalista deve ter. Também foi tema de estudo de Jung, o qual acreditava que a meditação era uma das formas de o sujeito acessar imagens arquetípicas; de Perls, que enfatizou a importância do aqui-e-agora. É também considerada pela abordagem transpessoal uma poderosa ferramenta para atingir outros níveis de consciência (Walsh \& Shapiro, 2006).

Mais recentemente, a meditação tem sido bastante pesquisada e empregada na linha da psicologia cognitivo-comportamental. Além de influenciar mudanças comportamentais, como a redução do estresse e ansiedade (Grossman, Niemannb, Schmidtc, \& Walachc, 2004), a meditação, assim como a psicologia cognitiva, caracteriza-se pela premissa fundamental de que a interpretação dos fatos é mais importante do que os fatos em si. Por esta razão, a prática meditativa tem sido descrita como uma forma de treinamento mental através da qual se busca educar a mente (Slagter et al., 2007).

De acordo com a literatura científica, a prática meditativa pode ser divida em duas formas principais: a) concentrativa - quando há o treino da atenção sobre um único foco, como a respiração, a contagem sincronizada à respiração, um mantra ou algum som, entre outros; sempre que houver uma distração, o praticante deve simplesmente retornar sua atenção ao foco; b) mindfulness - caracterizada pela consciência da experiência do momento presente, com uma atitude de aceitação, em que nenhum tipo de elaboração ou julgamento é utilizado. À medida que estímulos internos ou externos atingem a consciência do praticante, este simplesmente os observa e, assim como surgiram, deixa-os sumir, sem qualquer reflexão ou ruminação (Shapiro et al., 2005).

Segundo indica o estudo de Jha, Krompinger e Baime (2007), a meditação concentrativa está mais relacionada às funções de orientação e monitoramento, ao passo que a mindfulness tem maior correspondência ao estado de alerta. Na prática, estas duas formas caracterizam a meditação do tipo passivo - sentada e silenciosa - e podem ser empregadas em uma mesma sessão; entretanto, uma vez que a técnica mindfulness exige bastante treino, é mais comum que o praticante comece pelo tipo concentrativo, para só então conseguir a prática da atenção não engajada, como propõe a meditação do tipo mindfulness.

Os efeitos da meditação sentada e silenciosa vêm sendo amplamente investigados em diversos contextos, dos o campo da saúde mental e física é um dos principais. Quanto à cognição, por exemplo, estudos têm apontado que a prática meditativa pode influenciar positivamente alguns estilos de pensamento e sistemas cognitivos. Foi observado que o treino da meditação pode auxiliar na redução de pensamentos distrativos e ruminativos (Chambers, Yee Lo, \& Allen, 2008; Jain et al., 2007), além de propiciar um estilo cognitivo de maior aceitação (Bishop et al., 2004; Easterlin \& Cardeña, 1998). Ademais, a própria meditação pode constituir-se como uma estratégia de coping adaptativa, uma vez que se mostrou mais eficaz para o controle e redução de afetos negativos induzidos, em comparação à ruminação e à distração (Arch \& Craske, 2006; Broderick, 2005). Este padrão também foi evidenciado em uma pesquisa qualitativa, a qual detectou que os trabalhadores que participaram de uma intervenção com meditação para gerenciamento de estresse no trabalho relataram estratégias de coping mais positivas, como a percepção de maior autoeficácia e menor responsividade ao estresse (Walach et al., 2007).

Com relação aos sistemas cognitivos, tem sido observado que o treino da focalização da atenção, característico da prática meditativa, pode realmente auxiliar no controle desta função. Estudos utilizando tarefas comportamentais mostraram que o treino da meditação pode modular mecanismos atencionais e aumentar a capacidade do processamento de informação mediante um maior controle da distribuição dos recursos mentais (Carter et al., 2005; Slagter et al., 2007). Por meio deste tipo de tarefa também foi observado que a meditação do tipo mindfulness pode influenciar a capacidade de alerta e atenção sustentada (Chambers et al., 2008; Jha et al., 2007; Tang et al., 2007). Além disso, juntamente com a melhora no desempenho da atenção, também foi observada uma melhora estatisticamente significativa na memória de trabalho (Chambers et al., 2008) e nos escores de inteligência (Tang et al., 2007).

Estes dados, aliados a resultados que mostram uma relação entre a prática meditativa e uma maior autoconsciência (Brown \& Ryan, 2003; Chambers et 
al., 2008), ajudam a compreender por que a meditação tem sido chamada de técnica metacognitiva (Bishop et al., 2004). A cognição, contudo, não é um processo isolado, mas está em estreita relação com as emoções (Gross, 2002). Assim, é de esperar - e os dados apontam neste sentido - que, além de desempenhar um papel na regulação atencional, a meditação também pode influenciar a regulação emocional, sendo que estas respostas podem ser concomitantes e interdependentes (Brown \& Ryan, 2003; Chambers et al., 2008; Tang et al., 2007).

Foi observado que, juntamente com a redução da ruminação e melhora no desempenho atencional, os escores de afetos negativos diminuíram e os positivos aumentaram (Jain et al., 2007; Tang et al., 2007). Outro estudo mostrou que, após um programa utilizando meditação para pacientes que tiveram depressão, a chance de recaída era menor entre aqueles com maior consciência metacognitiva (Teasdale et al., 2002). Além disso, a maior autoconsciência em praticantes de mindfulness, medida através do Mindful Attention Awareness Scale (MAAS), associou-se a maior bem-estar psicológico (Brown \& Ryan, 2003).

Adicionalmente às repercussões nos domínios emocional e cognitivo, a meditação também pode influenciar a saúde física, como mostrou um estudo utilizando o programa Mindfulness-Based Stress Reduction (MBSR). Em comparação ao grupocontrole, os participantes da intervenção apresentaram maior ativação da área do cérebro relacionada ao bemestar, concomitantemente a uma melhor resposta imunológica após receber vacina para gripe (Davidson et al., 2003).

Além destes efeitos, a prática meditativa também parece ser uma experiência profunda, com repercussões em diversos âmbitos da vida. Entrevistas abertas indicam que muitos meditadores que buscam integrar sua prática ao seu ambiente profissional relatam melhor qualidade do trabalho, melhor tomada de decisões e melhor qualidade nas relações interpessoais, especialmente familiares (Duerr, 2004). Ademais, mulheres vítimas de violência que passaram por uma intervenção com meditação relataram sentimentos de bem-estar, maior conexão espiritual e melhores condições para lidar com as sequelas relacionadas ao abuso (Kane, 2006).

Todas estas pesquisas sugerem como a meditação pode repercutir positivamente na vida dos praticantes e ajudam a explicar por que ela tem sido tão associada à ciência psicológica. Não obstante, a maior parte dos dados é oriunda de questionários padronizados e os relatos subjetivos não abrangem amostras brasileiras.
Em vista disto, este estudo investigou a percepção de praticantes de meditação do tipo passivo (concentrativa e mindfulness) da Região Sul do Brasil acerca do impacto de sua prática no seu cotidiano. Embora existam particularidades que caracterizam as meditações concentrativa e mindfulness, muitas pesquisas também apontam resultados psicológicos comuns (Jha et al., 2007; Shapiro et al., 2005; Tang et al., 2007). Tendo-se como base estes achados e considerando-se o objetivo deste estudo, achou-se apropriado investigar ambos os tipos de meditação indiscriminadamente, sob a designação de meditação passiva - sentada e silenciosa.

\section{MÉTODO}

\section{Participantes}

Participaram deste estudo 105 praticantes de meditação do tipo passivo - sentada e silenciosa - com idade média de 41,4 anos $(D P=11,7)$, dos quais $64,8 \%$ eram do sexo feminino e $72,1 \%$ tinham terceiro grau completo. Os participantes, cujo tempo mínimo de prática deveria ser de um mês, frequentavam algum centro de meditação na cidade de Porto Alegre e foram selecionados por conveniência. Os centros compreenderam escolas de Yoga ou Budismo que ofereciam aulas e grupos de meditação.

\section{Instrumento}

Foi utilizado um questionário que contemplou dados sociodemográficos - como sexo, idade, estado civil, escolaridade, uso de medicação psicoativa e tratamento psicoterápico -, informações sobre a prática de meditação, como o tipo de meditação, o tempo, frequência e duração da prática, além de uma questão aberta em que se perguntava: "Como você acha que a meditação se reflete na sua vida?". A partir desta questão foi possível investigar a percepção dos praticantes sobre os efeitos de sua prática.

\section{Procedimentos}

Após a aprovação do projeto pelo Comitê de Ética em Pesquisa da Faculdade de Psicologia da UFRGS, foi realizado contato com os responsáveis pelos centros de meditação, com o intuito de explicar o objetivo do trabalho e os procedimentos a serem realizados. Após o consentimento do responsável e assinatura do "Termo de Concordância" da instituição, os praticantes eram contatados. Não houve recusa dos centros contatados $(n=10)$. Em grupo ou individualmente, os objetivos da pesquisa eram 
apresentados aos participantes e um Termo de Consentimento Livre e Esclarecido era entregue para que o lessem e, caso decidissem participar da pesquisa, o assinassem. Após o consentimento eram entregues envelopes contendo os instrumentos. As informações de preenchimento eram então fornecidas e era agendada uma data para a devolução dos envelopes. Foram entregues 112 envelopes, dos quais 105 retornaram (percentual de perdas ou recusas de $6,3 \%)$.

\section{Análise dos dados}

Os dados foram analisados por meio da análise de conteúdo (Bardin, 1977), de forma exploratória, uma vez que não se partiu de categorias previamente estabelecidas. A análise foi realizada a partir de três etapas principais: a pré-análise, a exploração do material e o tratamento dos resultados e interpretação. Na pré-análise, os conteúdos foram lidos e digitados. Na exploração do material, realizou-se a codificação, a qual passou por três fases: a) o recorte dos conteúdos em palavras ou expressõeschaves, constituindo-se nas unidades de análise; b) a enumeração das palavras, em que se priorizou a frequência de sua aparição; e c) a definição das categorias a partir de semelhanças e diferenças que representavam distintos temas. No tratamento dos resultados, a designação das palavras e expressões para cada categoria estipulada passou por um juiz, sendo avaliado o percentual de concordância através do índice Kappa, o qual resultou em $67 \%(p=0,003)$.

Com base nas respostas fornecidas, que compreenderam essencialmente adjetivos e/ou substantivos que descreviam os efeitos da meditação, optou-se pelo cálculo da frequência de respostas em cada categoria definida. Para as análises de fatores associados às categorias encontradas foi utilizada a regressão de Poisson (Barros \& Hirakata, 2003), em função de o desfecho ser a frequência de respostas apresentadas em cada categoria. Para a adequação dos dados foi utilizado o método de variância robusta, sendo estimadas as razões de prevalência (RP) e os seus respectivos intervalos de confiança de $95 \%$.

Para as análises ajustadas das distintas variáveis, seguiu-se um modelo hierárquico: no primeiro nível foram incluídas as variáveis sexo, idade, estado civil e a escolaridade; no segundo nível, a utilização de medicamentos com atividade psicoativa e a psicoterapia atual; e no terceiro nível foram incluídos o tempo de meditação em meses, a frequência semanal, a frequência diária e a duração em minutos de cada sessão. A inclusão/exclusão de variáveis no modelo foi definida por meio do teste de Wald de heterogeneidade para variáveis nominais e o de tendência para variáveis ordinais. As variáveis do primeiro e segundo níveis foram ajustadas entre si, sendo conservadas no modelo como possíveis fatores de confusão aquelas variáveis com nível de significância estatística $\leq 0,20$; para associação estatisticamente significativa utilizou-se o valor $p$ $\leq 0,05$. As variáveis do terceiro nível, por serem altamente colineares, foram ajustadas somente para as do primeiro e segundo níveis, conforme os critérios descritos. Todas as análises foram realizadas usando o programa estatístico STATA 9.0.

\section{RESULTADOS}

A Tabela 1 apresenta a caracterização sociodemográfica da amostra. Dos 105 participantes, $44,8 \%$ eram casados ou tinham um relacionamento estável, $21 \%$ faziam psicoterapia e $6,8 \%$ estavam sob uso de medicação psicoativa. O tempo de prática de meditação variou de um a 420 meses, com uma média de 91,8 meses $(D P=99,4)$, frequência média de quatro vezes por semana $(D P=2,1)$ e duração média de 35,4 minutos $(D P=19,2)$.

Tabela 1. Distribuição das Variáveis Sociodemográficas, Uso de Medicamento, Psicoterapia e Tempo de Meditação Entre os Participantes.

\begin{tabular}{|c|c|c|}
\hline Variáveis & $f$ & $\%$ \\
\hline \multicolumn{3}{|l|}{ Sexo } \\
\hline Masculino & 37 & $35,2 \%$ \\
\hline Feminino & 68 & $64,8 \%$ \\
\hline \multicolumn{3}{|l|}{ Idade (anos) } \\
\hline $20-29$ & 20 & $19,0 \%$ \\
\hline $30-39$ & 28 & $26,7 \%$ \\
\hline $40-49$ & 22 & $21,0 \%$ \\
\hline$\geq 50$ & 35 & $33,3 \%$ \\
\hline \multicolumn{3}{|l|}{ Estado civil } \\
\hline Casado/com companheiro & 47 & $44,8 \%$ \\
\hline Solteiro/sem companheiro & 58 & $55,2 \%$ \\
\hline \multicolumn{3}{|l|}{ Escolaridade (nível)* } \\
\hline Médio & 13 & $12,5 \%$ \\
\hline Superior incompleto & 16 & $15,4 \%$ \\
\hline Superior completo & 75 & $72,1 \%$ \\
\hline \multicolumn{3}{|c|}{ Uso de medicamento psicoativo* } \\
\hline Não & 96 & $93,2 \%$ \\
\hline Sim & 7 & $6,8 \%$ \\
\hline \multicolumn{3}{|l|}{ Psicoterapia } \\
\hline Não & 83 & $79,0 \%$ \\
\hline Sim & 22 & $21,0 \%$ \\
\hline \multicolumn{3}{|l|}{ Tempo de meditação* } \\
\hline Iniciante (até 12 meses) & 20 & $19,6 \%$ \\
\hline Intermediário (13-36 meses) & 25 & $24,5 \%$ \\
\hline Avançado (>36 meses) & 57 & $55,9 \%$ \\
\hline Total & 105 & \\
\hline
\end{tabular}

Notas: * Menos de 3\% de valores ignorados (missing) 
Quanto à questão aberta do questionário, foram levantadas 402 respostas, as quais foram categorizadas em seis dimensões: cognitiva, emocional, física, social, espiritual e outras, descritas a seguir: (a) a categoria cognitiva incluiu respostas relativas a aspectos mentais oriundos da prática de meditação, como maior autoconhecimento, maior clareza e compreensão de si e das coisas, maior capacidade de atenção e concentração, objetividade, centramento, e sentimento de êxito na utilização de estratégias de coping; (b) a categoria emocional incluiu respostas de cunho afetivo, como sentimentos de tranquilidade, paz, equilíbrio emocional, redução do estresse e ansiedade, bem-estar e qualidade de vida, além de mais autoestima e segurança; (c) a categoria física envolveu respostas como relaxamento do corpo e melhor qualidade do sono e da saúde em geral; (d) a categoria social abrangeu aspectos interpessoais, como maior aceitação dos outros e sensação de pertença a um grupo; e (e) a categoria espiritual incluiu respostas representando aspectos de ordem superior, como um sentimento de conexão com algo maior, aproximação de Deus e equilíbrio espiritual. As categorias e suas frequências, além das respostas com maior aparição em cada categoria, estão apresentadas na tabela 2.

Tabela 2. Percentuais das Respostas Mais Freqüentes por Categoria

\begin{tabular}{lcc}
\hline Categorias & $\boldsymbol{f}$ & $\mathbf{\%}$ \\
\hline Cognitiva & $\mathbf{1 8 8}$ & $\mathbf{4 6 , 7 \%}$ \\
Mais atenção, concentração e foco & 29 & $15,4 \%$ \\
Mais clareza mental & 24 & $12,7 \%$ \\
Mais consciência de si, dos outros e das coisas & 18 & $9,5 \%$ \\
Autoconhecimento & 17 & $9,0 \%$ \\
Melhora no enfrentamento de problemas & 15 & $7,9 \%$ \\
Mais centrada/o & 13 & $6,9 \%$ \\
Outras respostas & 72 & $38,6 \%$ \\
Emocional & $\mathbf{1 3 5}$ & $\mathbf{3 3 , 5 \%}$ \\
Calma e tranquilidade & 32 & $23,7 \%$ \\
Equilíbrio emocional & 16 & $11,8 \%$ \\
Mais feliz e maior bem-estar & 14 & $10,3 \%$ \\
Menos estresse e ansiedade & 13 & $9,6 \%$ \\
Paz & 12 & $8,8 \%$ \\
Outras respostas & 48 & $35,8 \%$ \\
Física & $\mathbf{3 3}$ & $\mathbf{8 , 2} \%$ \\
Relaxamento físico & 13 & $39,3 \%$ \\
Mais energia e disposição & 9 & $27,2 \%$ \\
Melhora na qualidade da saúde e do sono & 7 & $21,2 \%$ \\
Outras respostas & 4 & $12,3 \%$ \\
Social & $\mathbf{2 1}$ & $\mathbf{5 , 2} \%$ \\
Fortalece e melhora relacionamentos & 8 & $38,1 \%$ \\
Mais aceitação e receptividade nos relacionamentos & 7 & $33,3 \%$ \\
Outras respostas & 6 & $28,6 \%$ \\
Espiritual & $\mathbf{1 5}$ & $\mathbf{3 , 7} \%$ \\
Sentimento de aproximação e conexão com ser divino & 6 & $40 \%$ \\
Dedicação a Deus & 3 & $20 \%$ \\
Outras respostas & 6 & $40 \%$ \\
Outras & $\mathbf{1 0}$ & $\mathbf{2 , 4} \%$ \\
Total de respostas de todas categorias & $\mathbf{4 0 2}$ & $\mathbf{1 0 0 \%}$ \\
\hline & & \\
& &
\end{tabular}

É importante ressaltar que as análises contemplaram o total de respostas $(n=402)$ e não o total de pessoas, visto que cada participante podia apresentar mais de uma resposta, inclusive em uma mesma categoria. A frequência com que as palavras e/ou expressões mencionadas por uma pessoa apareceram em uma mesma categoria variou de uma a seis vezes. Assim, também foi possível observar a porcentagem de sujeitos conforme o número de respostas obtidas em cada categoria (Figura 1). A maioria das pessoas relatou algum benefício cognitivo $(83,8 \%)$ e emocional $(77,1 \%)$ pelo menos uma vez. Com relação às outras categorias, apenas $23,8 \%$ mencionaram benefícios físicos, $15,2 \%$ mencionaram benefícios sociais e 13,3\%, benefícios espirituais.

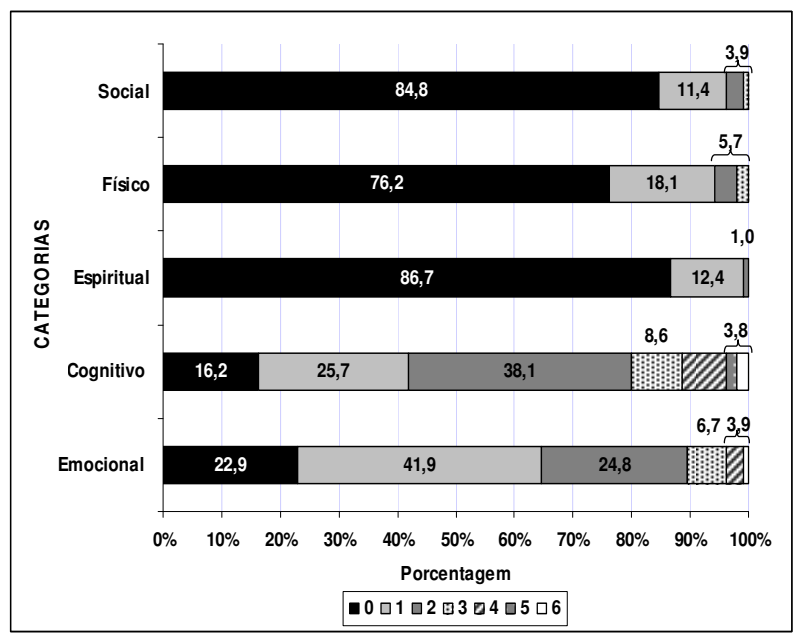

Figura 1. Porcentagem de Participantes Conforme a Frequência de suas Respostas em Cada Categoria

Nas Tabelas 3, 4 e 5 estão incluídas as análises ajustadas para cada uma das categorias, as quais foram realizadas por níveis de hierarquia, conforme descrito no método. Observou-se que o número médio de vezes em que as respostas da categoria emocional foram mencionadas foi $65 \%$ mais frequente entre os que realizam psicoterapia em comparação com os que não a realizam $(p=0,006)$. Da mesma forma, houve uma associação direta significativa entre o tempo de meditação e a média de vezes em que as respostas da categoria emocional apareceram (RP iniciante; intermediário 1,78; avançado 1,$69 ; p=0,04)$. Nenhuma das outras exposições mostrou associação significativa com este desfecho. Para a categoria cognitiva, houve uma associação com escolaridade, ou seja, foi observado um maior número de respostas nesta categoria entre os participantes com ensino superior completo $(p=0,02)$.

Nenhum iniciante apresentou respostas quanto à categoria espiritual. Entre os praticantes que 
mencionaram este tipo de benefício, observou-se uma associação positiva com a frequência semanal e a duração da sessão (incremento de $31 \%$ para cada dia e de $2 \%$ para cada minuto de meditação; valores $p=0,05$ e $p=0,03$ respectivamente).
As respostas de bem-estar físico foram $66 \%$ menos frequentes entre as pessoas com mais de 50 anos, quando comparadas com o grupo de 20 a 29 anos $(p=0,04)$. Nenhuma das variáveis independentes esteve associada significativamente com a categoria social.

Tabela 3. Resultados da Análise Ajustada da Regressão de Poisson das Categorias Emocional, Cognitiva, Espiritual, Física e Social com as Variáveis Sociodemográficas (nível 1)

\begin{tabular}{|c|c|c|c|c|c|}
\hline & Emocional & Cognitiva & Espiritual & Física & Social \\
\hline & Análise ajustada & Análise ajustada & Análise ajustada & Análise ajustada & Análise ajustada \\
\hline & RP (IC95\%) & RP (IC95\%) & RP (IC95\%) & RP (IC95\%) & RP (IC95\%) \\
\hline $1^{\mathrm{a}}$ Sexo & $P=0,2 \#$ & $P=0,8 \#$ & $P=0,9 \#$ & $P=0,6^{*}$ & $P=0,1^{*}$ \\
\hline Masculino & 1,00 & 1,00 & 1,00 & 1,00 & 1,00 \\
\hline Feminino & $0,79(0,57-1,11)$ & $0,97(0,71-1,32)$ & $0,95(0,35-2,59)$ & $0,83(0,39-1,74)$ & $2,31(0,77-6,90)$ \\
\hline Idade (anos) & $P=0,3 \#$ & $P=0,7 \#$ & $P=0,4 \#$ & $P=0,04^{*}$ & $P=1,0 \#$ \\
\hline $20-29$ & 1,00 & 1,00 & 1,00 & 1,00 & 1,00 \\
\hline $30-39$ & $1,18(0,75-1,88)$ & $0,88(0,58-1,33)$ & $0,68(0,18-2,53)$ & $0,36(0,10-1,23)$ & $0,59(0,16-2,21)$ \\
\hline $40-49$ & $1,05(0,68-1,58)$ & $0,99(0,63-1,57)$ & $0,19(0,02-1,80)$ & $1,09(0,41-2,88)$ & $0,71(0,12-4,37)$ \\
\hline$\geq 50$ & $0,87(0,58-1,34)$ & $0,88(0,58-1,33)$ & $0,63(0,21-1,93)$ & $0,34(0,12-0,99)$ & $0,86(0,25-2,91)$ \\
\hline Estado civil & $P=0,9 \#$ & $P=0,6 \#$ & $P=0,2 \#$ & $P=0,3^{*}$ & $P=0,9 *$ \\
\hline Com companheiro & 1,00 & 1,00 & 1,00 & 1,00 & 1,00 \\
\hline Sem companheiro & $1,03(0,75-1,43)$ & $1,07(0,81-1,41)$ & $0,54(0,20-1,49)$ & $1,46(0,69-3,10)$ & $1,15(0,41-3,23)$ \\
\hline Escolaridade (nível) & $P=0,3 \#$ & $P=0,02 \#$ & $P=0,4 \#$ & $P=0,7 \#$ & $P=0,3 \#$ \\
\hline Médio & 1,00 & 1,00 & 1,00 & 1,00 & 1,00 \\
\hline Superior incompleto & $1,42(0,74-2,75)$ & $1,75(0,94-3,27)$ & $0,24(0,03-2,30)$ & $1,29(0,35-4,72)$ & $0,54(0,13-2,26)$ \\
\hline Superior completo & $1,44(0,81-2,58)$ & $1,95(1,13-3,37)$ & $0,46(0,13-1,53)$ & $1,29(0,45-3,74)$ & $0,45(0,12-1,62)$ \\
\hline
\end{tabular}

Notas:* Teste de Wald de heterogeneidade

\# Teste de Wald de tendência

Tabela 4. Resultados da Análise Ajustada da Regressão de Poisson das Categorias Emocional, Cognitiva, Espiritual, Física e Social com as Variáveis de Tratamento (nível 2)

\begin{tabular}{|c|c|c|c|c|c|}
\hline & \\
\hline & Emocional & Cognitiva & Espiritual & Física & Social \\
\hline & Análise ajustada & Análise ajustada & Análise ajustada & Análise ajustada & Análise ajustada \\
\hline & RP (IC95\%) & RP (IC95\%) & RP (IC95\%) & RP (IC95\%) & RP (IC95\%) \\
\hline $2^{\mathrm{a}}$ Medicamentos psicoativos & $P=1,0 \#$ & $P=0,4 \#$ & $P=1,0 \#$ & $P=1,0 *$ & $P=0,7 *$ \\
\hline Não & 1,00 & 1,00 & 1,00 & 1,00 & 1,00 \\
\hline Sim & $0,94(0,50-1,75)$ & $0,79(0,43-1,43)$ & $1,03(0,17-6,10)$ & $0,96(0,17-5,28)$ & $1,51(0,23-9,80)$ \\
\hline Psicoterapia & $P=0,006 \#$ & $P=0,5 \#$ & $P=0,9 \#$ & $P=1,0 *$ & $P=0,7 *$ \\
\hline Não & 1,00 & 1,00 & 1,00 & 1,00 & 1,00 \\
\hline Sim & $1,65(1,15-2,36)$ & $0,87(0,56-1,36)$ & $0,94(0,28-3,21)$ & $1,00(0,41-2,44)$ & $0,74(0,13-4,16)$ \\
\hline
\end{tabular}

Notas: * Teste de Wald de heterogeneidade \# Teste de Wald de tendência

a) ajustado para variáveis do nível 1 com valor $\mathrm{p} \leq 0,20$

Tabela 5. Resultados da Análise Ajustada da Regressão de Poisson das Categorias Emocional, Cognitiva, Espiritual, Física e Social com as Variáveis de Tempo de Meditação (nível 3)

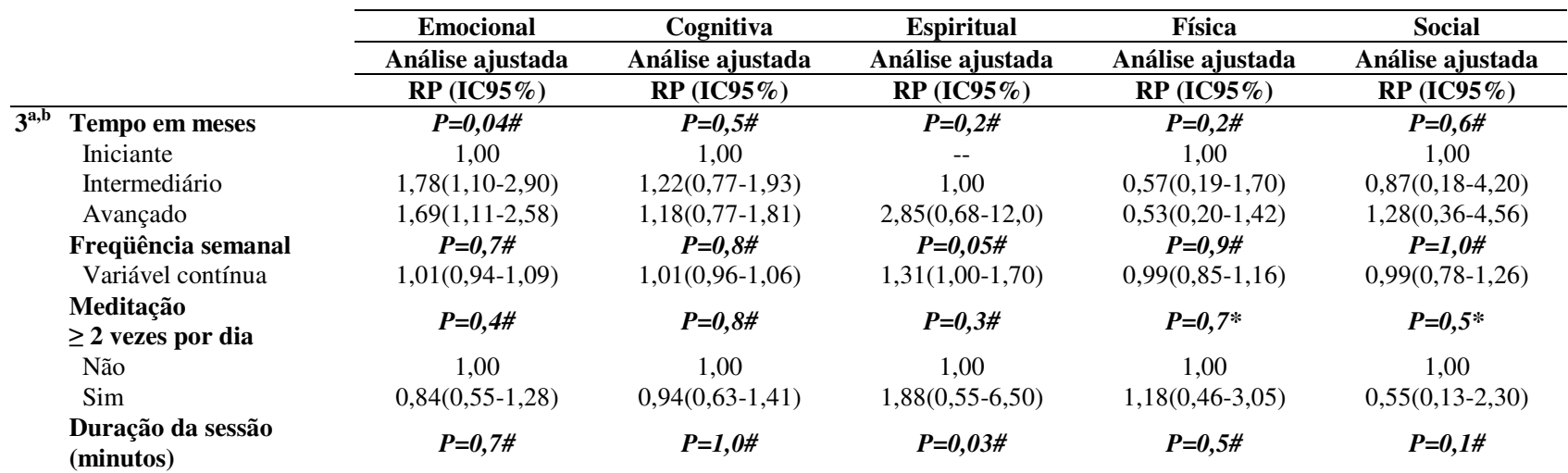

Variável contínua

$1,00(0,99-1,01) \quad 1,00(0,99-1,01)$

$1,02(1,00-1,03)$

$0,99(0,98-1,01)$

$1,01(1,00-1,03)$

Notas: * Teste de Wald de heterogeneidade \# Teste de Wald de tendência

a) ajustado para variáveis do nível 1 com valor $\mathrm{p} \leq 0,20$

b) ajustado para variáveis do nível 2 com valor $\mathrm{p} \leq 0,20$ 


\section{DISCUSSÃO}

Afinal, por que meditar? As respostas obtidas na pergunta aberta sobre como os praticantes percebem que a meditação se reflete nas suas vidas indicam que eles experimentam e mantêm a prática meditativa porque sentem uma série de resultados positivos, que abrangem diferentes âmbitos de sua vida. Foram citados benefícios emocionais, cognitivos, físicos, espirituais e sociais, porém predominaram as respostas relacionadas às áreas cognitiva e emocional, as quais apresentaram percentuais mais altos, corroborando estudos anteriores (Chambers et al., 2008; Jain et al., 2007; Tang et al., 2007).

Com a análise ajustada da regressão de Poisson também foi possível observar alguns fatores associados às categorias obtidas. Os benefícios emocionais, por exemplo, foram mais mencionados por quem faz psicoterapia. Este dado apoia as observações que apontam um efeito mútuo entre meditação e tratamento psicoterápico no que tange à regulação emocional (Hayes \& Feldman, 2004). Além disso, a associação entre os efeitos emocionais da meditação e a psicoterapia corrobora a ideia de que ambas as técnicas compartilham características centrais, como a busca da redução do sofrimento por um processo de refinamento da consciência e de desidentificação com processos mentais disfuncionais (Walsh \& Shapiro, 2006).

Não obstante, pesquisas têm mostrado que a meditação, por si só, pode repercutir na vida afetiva do praticante, ainda que com intervenções breves (Arch \& Craske, 2006; Tang et al., 2007). Em nossa pesquisa, tanto praticantes iniciantes ou intermediários como avançados relataram benefícios emocionais; porém foi encontrada uma associação significativa entre o tempo de prática e estas respostas, cuja prevalência foi maior entre as pessoas que praticavam meditação havia mais de um ano. Este achado está em consonância com dois estudos que encontraram escores de experiência emocional positiva significativamente maiores entre meditadores com mais de 12 (Easterlin \& Cardeña, 1998) e 18 meses de prática (Aftanas \& Golocheikine, 2001). A influência do tempo de prática pode ser importante, visto que a regulação emocional é um processo que envolve a aprendizagem de como ampliar, atenuar ou manter a força das reações emocionais (Gross, 2002). Assim, quanto maior for o treino em meditação, maior poderá ser a capacidade do indivíduo de se autorregular positivamente $\mathrm{e}$, possivelmente, de forma mais automática, sem estar, necessariamente, consciente do processo. Além disso, conforme demonstraram
Davidson et al. (2003), a meditação pode ativar áreas cerebrais associadas ao bem-estar. Assim, considerando-se a plasticidade dos circuitos emocionais, sugere-se que a quantidade de práticas pode associar-se à magnitude destes efeitos cerebrais, gerando padrões comportamentais mais estáveis a partir deles.

Já para a categoria cognitiva, observou-se associação com a escolaridade, especificamente com o terceiro grau completo. Este dado sugere que, assim como a psicoterapia pode reforçar os efeitos emocionais da meditação, possivelmente ajudando o praticante a conectar-se melhor com a experiência, o nível de instrução do meditador pode ser importante para que ele consiga dar mais sentido ao processo e seus efeitos cognitivos, bem como usufruir melhor destes. Além disso, os centros de meditação comumente oferecem atividades complementares, como retiros e palestras, a fim de que o praticante tenha uma maior compreensão dos preceitos que sustentam a prática de meditação. Embora este dado não tenha sido relatado na seção de resultados, a maioria das pessoas investigadas informou fazer algum tipo de atividade complementar e relacionada, como leituras, palestras e retiros. Dessa forma, outra hipótese é que um nível de instrução maior pode facilitar a integração da vivência pessoal da meditação com os postulados transmitidos.

Também se sugere que é nesta fase de envolvimento e aprofundamento dos preceitos filosóficos que, para alguns meditadores, pode começar a ocorrer uma abertura à dimensão espiritual da prática, já que neste estudo apenas os meditadores intermediários e avançados mencionaram benefícios da categoria espiritual. Segundo um levantamento realizado em uma amostra norte-americana, quando a busca da meditação não se deve exclusivamente a um desejo de afiliação a suas tradições filosóficas ou religiosas, há três razões principais que a motivam: os benefícios clínicos; a ideia de que a meditação é uma porta para a autoexploração profunda; e a intenção de transformação pessoal e social (Duerr, 2004). Da mesma forma, estas razões parecem caracterizar a motivação da amostra investigada em nosso estudo. Além do grande número de respostas emocionais e cognitivas e do fato de nenhum iniciante ter mencionado algum benefício espiritual, em geral a frequência das respostas da categoria espiritual foi baixa. Assim, é possível que a espiritualidade não seja o objetivo inicial para muitos praticantes, mas que com o aprofundamento e a continuidade da prática algumas pessoas naturalmente comecem a voltar-se e abrir-se para a tradição espiritual na qual se originou o 
tipo de meditação que está realizando. Ainda para esta categoria, a pequena associação encontrada com os minutos de prática $(2 \%)$ pode sugerir que tal resultado se deva ao acaso. Já a associação encontrada entre os benefícios espirituais e a frequência semanal pode indicar que são exatamente os praticantes que frequentam os centros de meditação mais vezes na semana aqueles que se envolvem com os postulados filosóficos ou espirituais da prática.

As respostas fornecidas neste estudo e suas respectivas categorias possibilitaram identificar como os praticantes percebem os efeitos de sua prática, os quais, com exceção dos efeitos físicos, parecem independer da idade e do sexo. Para a categoria física observou-se uma associação com a idade, visto que as respostas foram significativamente menos frequentes entre as pessoas com mais de 50 anos. Uma hipótese para este achado é que na faixa etária mais avançada há um enfraquecimento natural do vigor e da qualidade da saúde em comparação com os mais jovens, de forma que, ao compará-los, esta categoria de benefícios aparece menos entre os mais velhos.

Além das associações observadas entre as categorias e algumas variáveis incluídas no modelo hierárquico, outra hipótese, baseada na semelhança entre os dados do presente estudo e os de Duerr (2004) é que a experiência da meditação entre os ocidentais talvez independa de sua cultura. Das 24 palavras e expressões-chaves que entrevistados norteamericanos apontaram como qualidades cultivadas através de sua prática (Duerr, 2004), 11 também apareceram nas respostas dos praticantes de Porto Alegre: maior consciência, clareza, calma, concentração, foco, atenção plena, autoconhecimento, bem-estar, aceitação, senso de interconexão e menor ansiedade.

A semelhança destas respostas e a frequência das categorias obtidas no presente estudo indicam que, a despeito da cultura ocidental, e até mesmo da influência dos valores cultivados pelas tradições que originaram a prática meditativa, a técnica da meditação pode ter seus efeitos mediados por alguns processos psicológicos que são característicos da natureza humana, como a metacognição e a regulação emocional. Além disso, a alta porcentagem de pessoas que mencionaram efeitos cognitivos e emocionais $(83,8 \%$ e $77,1 \%$, respectivamente) apoia a ideia de que a meditação estimula estes dois processos de forma interdependente (Bishop et al., 2004; Broderick, 2005; Chambers et al., 2008). Adicionalmente, é possível que para alguns participantes, os benefícios cognitivos e emocionais também estejam interligados com as outras categorias citadas, como melhor saúde física, melhor convívio social e bem-estar espiritual.

\section{CONSIDERAÇÕES FINAIS}

Embora os objetivos do estudo tenham sido alcançados, contribuindo para uma maior compreensão da percepção dos praticantes acerca dos efeitos da prática da meditação nas suas vidas, podemos apontar algumas limitações desta pesquisa. Uma delas é a causalidade reversa, ou seja, as associações encontradas a partir do delineamento transversal não permitem conclusões sobre relações de causa e efeito. Outra limitação foi o tamanho pequeno da amostra, o qual possivelmente interferiu no poder do estudo. Mais estudos sobre os efeitos da meditação, especialmente experimentais e longitudinais, com amostras maiores, podem contribuir neste sentido.

Não obstante, a partir dos relatos subjetivos dos participantes, pode-se concluir que a experiência da meditação se reflete positivamente em diferentes domínios da vida do praticante. Para as pessoas que mencionaram mais de um tipo de benefício, é possível que tais efeitos estejam atuando por meio de um mecanismo de retroalimentação. Além disso, com base na predominância de benefícios cognitivos e emocionais, sugere-se que a prática meditativa pode se constituir como uma ferramenta útil à Psicologia, concorrendo para um desenvolvimento psicológico saudável.

\section{REFERÊNCIAS}

Aftanas, L. I., \& Golocheikine, S. A. (2001). Human anterior and frontal midline theta and lower alpha reflect emotionally positive state and internalized attention: High-resolution EEG investigation of meditation. Neuroscience Letters, 310, 57-60.

Arch, J. J., \& Craske, M. G. (2006). Mechanisms of mindfulness: Emotion regulation following a focused breathing induction. Behavior Research and Therapy, 44, 1849-1858.

Bardin, L. (1977). Análise de conteúdo. Lisboa: Edições 70.

Barros, A. J. D., \& Hirakata, V. N. (2003). Alternatives for logistic regression in cross-sectional studies: An empirical comparison of models that directly estimate the prevalence ratio. BMC Medical Research Methodology, 3. Recuperado em 06 de setembro de $2008 \mathrm{em}$ http://www.biomedcentral.com/bmcmedresmethodol/

Bishop, S. R., Lau, M., Shapiro, S., Carlson, L., Anderson, N. D., Carmody, J., Segal, Z. V., Abbey, S., Velting, D., \& Devins, G. (2004). Mindfulness: A proposed operational definition. Clinical Psychology: Science and Practice, 11(3), 230-241.

Broderick, P. C. (2005). Mindfulness and coping with dysphoric mood: Contrasts with rumination and distraction. Cognitive Therapy and Research, 29(5), 501-510. 
Brown, K. W., \& Ryan, R. M. (2003). The benefits of being present: Mindfulness and its role in psychological well-being. Journal of Personality and Social Psychology, 84(4), 822-848.

Carter, O. L., Presti, D. E., Callistemon, C., Ungerer, Y., Liu, G. B., \& Pettigrew, J. D. (2005). Meditation alters perceptual rivalry in Tibetan Buddhist monks. Current Biology, 15, 412-413.

Chambers, R., Yee Lo, B. C., \& Allen, N. B. (2008). The impact of intensive mindfulness training on attentional control, cognitive style, and affect. Cognitive Therapy and Research, 32, 303-322.

Davidson, R. J., Kabat-Zinn, J., Schumacher, J., Rosenkranz, M., Muller, D., Santorelli, S. F., Urbanowski, F., Harrington, A., Bouns, K., \& Sheridan, J. F. (2003). Alterations in brain and immune function produced by mindfulness meditation. Psychosomatic Medicine, 65, 564-570.

Duerr, M. (2004). A powerful silence: the role of meditation and other contemplative practices in American life and work. Recuperado em 20 de Julho de 2008 em http://www.contemplativemind.org.

Easterlin, B. L., \& Cardeña, E. (1998). Cognitive and emotional differences between short- and long-term vipassana meditators. Imagination, Cognition and Personality, 18(1), 69-81.

Gross, J. J. (2002). Emotion regulation: affective, cognitive, and social consequences. Psychophysiology, 39, 281-291.

Grossman, P., Niemannb, L., Schmidtc, S., \& Walachc, H. (2004). Mindfulness-based stress reduction and health benefits: A metaanalysis. Journal of Psychosomatic Research, 57, 35-43.

Hayes, A. M., \& Feldman, G. (2004). Clarifying the construct of mindfulness in the context of emotion regulation and the process of change in therapy. Clinical Psychology: Science and Practice, 11(3), 255-262.

Jain, S., Shapiro, S. L., Swanick, S., Roesch, S. C., Mills, P. J., Bell, I., \& Schwartz, G. E. R. (2007). A randomized controlled trial of mindfulness meditation versus relaxation training: Effects on distress, positive states of mind, rumination and distraction. Annals of Behavioral Medicine, 33(1), 11-21.

Jha, A. P., Krompinger, J., \& Baime, M. (2007). Mindfulness training modifies subsystems of attention. Cognitive, Affective, \& Behavioral Neuroscience, 7(2), 109-119.
Kane, K. E. (2006). The phenomenology of meditation for female survivors of intimate partner violence. Violence Against Women, 12(5), 501-518.

Shapiro, S. L., Schwartz, G. E., \& Santerre, C. (2005). Meditation and positive psychology. In C. R. Snyder \& S. J. Lopez (Eds.), Handbook of positive psychology (pp. 632-645). New York: Oxford USA Trade.

Slagter, H. A., Lutz, A., Greischar, L. L., Francis, A. D., Nieuwenhuis, S., Davis, J. M., \& Davidson, R. J. (2007). Mental training affects distribution of limited brain resources. PLoS Biology, 5. Recuperado em 10 de fevereiro de 2008 em http://www.plosbiology.org

Tang, Y., Ma, Y., Wang, J., Fan, Y., Feng, S., Lu, Q., Yu, Q., Sui, D., Rothbart, M. K., Fan, M., \& Posner, M. I. (2007). Short-term meditation training improves attention and self-regulation. Proceedings of the National Academy of Sciences, 104(43), 17152-17156.

Teasdale, J. D., Moore, R. G., Hayhurst, H., Pope, M., Williams, S., \& Segal, Z. V. (2002). Metacognitive awareness and prevention of relapse in depression: empirical evidence. Journal of Consulting and Clinical Psychology, 70(2), 275-287.

Walach, H., Nord, E., Zier, C., Dietz-Waschkowski, B., Kersig, S., \& Schüpbach, H. (2007). Mindfulness-based stress reduction as a method for personnel development: A pilot evaluation. International Journal of Stress Management, 14(2), 188-198.

Walsh, R., \& Shapiro, S. L. (2006). The meeting of meditative disciplines and western psychology: A mutually enriching dialogue. American Psychologist, 61(3), 227-239.

Recebido em 23/10/2008 Aceito em 06/02/2009

Endereço para correspondência : Carolina Baptista Menezes. Rua Ramiro Barcelos, 2600, sala 115, Bairro Santa Cecília, CEP 90035-003, Porto Alegre-RS, Brasil. E-mail: menezescarolina@ hotmail.com 